\title{
Towards Online Adaptive Ambient Intelligent Environments for Multiple Occupants
}

\author{
Hani Hagras \\ School of Computer Science and Electronic Engineering, University of Essex, UK \\ hani@essex.ac.uk
}

Adaptation is a relationship between a system and its environment where change is provoked to facilitate the survival of the system in the environment. Biological systems exhibit different types of adaptation so as to regulate themselves and change their structure as they interact with the environment.

The dynamic and ad-hoc nature of Ambient Intelligent Environments (AIEs) means that the environment has to adapt to changing operating conditions and user changing preferences and behaviours and to enable more efficient and effective operation while avoiding any system failure. Thus there is a need to provide autonomous intelligent adaptive techniques which should be able to create models which could be evolved and adapted online in a life learning mode. These models need to be transparent and easy to be read and interpreted via the normal user to enable the user to better analyze the system and its performance. These intelligent systems should allow to control the environment on the user behalf and to his satisfaction to perform given tasks. The intelligent approaches used should have low computational overheads to effectively operate on the embedded hardware platforms present in the everyday environments which have small memory and processor capabilities. These models need to be transparent and easy to be ready and interpreted via the normal user to enable the user to better analyze the system and its performance. This task based system could be used to control the environment on the user behalf and to his satisfaction to perform given tasks. In addition, the intelligent approaches should allow for real-time data mining of the user data and create on-the-fly updateable models of the user preferences that could be executed over the pervasive network. Moreover, there is a need to provide an adaptive life-long learning mechanism that will allow the system to adapt to the changing environmental and user preferences over short and long term intervals. There is a need also to provide robust mechanisms that will allow handling the varying and unpredictable conditions associated with the dynamic environment and user preferences.

This talk will present novel adaptation strategies that will allow the environments to adapt to the uncertainties associated with the changes in the environments characteristics, context as well as changes in the user(s) preferences in AIEs. The talk will present new general type-2 fuzzy logic systems that could be used to model and handle the uncertainties associated with these environments where we will present real world experiments from the Essex iSpace real world AIE. 